\title{
ATENCIÓN TEMPRANA A LAS NECESIDADES DEL NIÑO
}

\author{
Paola Sirna Argumedo
}

\begin{abstract}
RESUMEN
Es necesario observar a los niños desde muy pequeños y brindarles una atención oportuna a las dificultades que vaya presentando, ya sea en las áreas: emocional, dándole amor y seguridad; motora; de lenguaje 0 sensorial. Dicha atención temprana pueden ayudar a que el pequeño supere su desfase temporal y no desencadene en otras dificultades que no le ayuden a desarrollarse $y / 0$ desenvolverse favorablemente en la escuela.
\end{abstract}

\section{PALABRAS CLAVE}

Atención oportuna, amor, seguridad, desarrollo, escuela.

\section{ABSTRACT}

It is necessary to observe the children from very young, and to provide them a timely care to the difficulties that it is presenting, either in the areas: emotional, giving them love and security; motorboat; language or sensory. Such early attentions can help the child to overcome his time lag and not to trigger other difficulties that do not help them develop and/or develop favorably in school.

\section{KEYWORDS}

Timely care, love, security, development, school.
$\mathrm{D}$ urante mis años de estudio en educación, siempre escuché a diferentes maestros decir que mientras más pronto se le detectara alguna dificultad a un pequeño y se la tratara, éste tenía mayor posibilidad de superar dicha dificultad. Y qué pasa si detectas algo diferente a muy temprana edad, entre uno a dos años, cómo saber si es parte de su propio ritmo de aprendizaje, si es parte de su maduración cognitiva o si hay de por medio alguna dificultad que no le permita desarrollarse como la mayoría de los niños, sobre todo, en el futuro. Todos los padres anhelan para sus hijos lo mejor y tal vez inconscientemente desean que sus niños logren todo lo que ellos no pudieron lograr, es una gran responsabilidad y carga para un pequeño poder cubrir esas expectativas; y qué pasa si no las supera, cómo hacerle entender al padre de familia que su niño es único, que su niño es diferente a otros pequeños, ni mejor, ni peor que nadie, sólo alguien especial, con su propia forma de ser, de sentir, de aprender y con su propia forma de reaccionar ante diferentes situaciones. En todos mis años ejerciendo mi carrera en educación inicial he visto diferentes casos donde los padres se preocupan mucho cuando sus pequeños no logran realizar la acción que deberían de lograr de acuerdo a la edad o el mes en elque están.

En el primer año de vida, los pediatras van observando los logros de cada bebé, midiendo su talla, el contorno de la cabeza y pesándolos, preguntando a los padres si el bebé realizó la acción que le correspondería realizar de acuerdo a su edad, o si responde a su nombre, a los sonidos, entre otras cosas, ya que tienen claro que si hay un retraso en el área motora o de lenguaje podría indicar que algo no está del todo bien en el pequeño; pero, cuándo hablamos de un retraso en el lenguaje, para los pediatras es primordial preguntar si el pequeño responde a diferentes estímulos de su entorno porque lo primero que los niños desarrollan es el lenguaje 
comprensivo, este es muy necesario para desarrollar otras capacidades, por lo que siempre que surge un leve desfase en esta área, primero descartan que sea algún problema físico.

Los terapistas de lenguaje hablan de un retraso en el lenguaje recién a partir de los tres años de edady muchos sugieren que a partir de los dos años y medio, si aún no tiene un lenguaje oral, el pequeño debería llevar una terapia de lenguaje que lo ayude a superar este dilema. Pero cómo puede un padre de familia, en especial quien tiene su primer hijo, tener la paciencia y la serenidad para poder esperar con calma mes a mes y darle toda la atención y ayuda necesaria a su niño para que éste pueda sobrellevar o superar tal dificultad. Como madre creo que sólo el amor puede ayudar a sobrellevar dicha angustia y darlo todo por nuestros pequeños, nuestro tiempo, nuestro cariño, nuestras enseñanzas, para que ellos puedan superar pronto tal dificultad. Y si aún así el pequeño no puede superar dicho problema, cómo hacer que un padre pueda esperar recién hasta los seis o siete años de edad de su hijo para poder tener un diagnóstico más acertado de la dificultad que tiene su pequeño, por lo general a partir de esa edad recién se le puede hacer una evaluación más detallada o mejor dicho se le puede dar un nombre a su problema. Y si en lugar del lenguaje, o el área motora, la dificultad del niño es sensorial, cómo repercute esto en su desenvolvimiento académico cuando esté en primaria, si no es tratado a tiempo.

\section{Proteger la imagen personal del niño}

Desde los primeros años de vida, la persona va formando poco a poco una imagen de sí mismo, el cual será la base para construir una autoestima adecuada, los padres o cuidadores deben de tener mucho cuidado en cómo educan o corrigen a sus hijos, ya que de eso depende el desenvolvimiento del pequeño y su triunfo o fracaso en la escuela, entre otros ámbitos. Si en estos primeros años de vida se van observando pequeñas dificultades es necesario tratarlas a la brevedad posible, sin alarmarse y de manera de juego, ayudándonos con diferentes profesionales, dependiendo de la necesidad del pequeño, para que no ocasione mayores dificultades en el futuro. Lo peor que se puede hacer, es comparar a los niños con otros pequeños de su edad o hacerle sentir aunque sea de forma indirecta tristeza, impotencia o en muchos casos, decepción, porque los niños pueden sentirlo y de alguna manera va influir negativamente en ellos esas actitudes.

Según el artículo de Guía Infantil (2013), de acuerdo al grado de autoestima, dependerá el desarrollo del aprendizaje del niño, sus buenas relaciones con las personas de su entorno y/o actividades y la construcción de su felicidad. Si los padres y maestros manejan adecuadamente este aspecto en el niño, el pequeño va a sentirse competente, seguro y valioso, podrá entender que es importante aprender y no se va a sentir disminuido cuando necesite ayuda.

Según Russek S. (2007), una baja autoestima afecta todas las áreas de la vida de un niño, provocando sentimientos de angustia, temor, vergüenza, indecisión, impotencia, apatía, culpa, tristeza y/o depresión. El pequeño con baja autoestima se compara constantemente con los éxitos de los demás y por lo general su conclusión es que los demás son superiores a él. Por lo que se vuelve dependiente de la opinión de terceros, baja sus ganas de tomar la iniciativa y realizar algo por sí mismo, ya que existe el temor al fracaso; y con el tiempo, puede volverse una persona envidiosa que busque constantemente defectos en los demás para no sentirse mal consigo mismo.

\section{El desarrollo del niño}

Los seres humanos recibimos en primer lugar una herencia genética, la cual es el punto de partida para nuestro desarrollo, este se complementa con la estimulación que recibimos del medio ambiente que nos rodea.

Según Sarmiento, B. y Marín, J. (2009), el desarrollo desde el punto de vista piagetiano es netamente biológica, es el proceso de cambio de una estructura orgánica simple a una compleja, es una estructura equilibrada, porque tiene la capacidad de satisfacer necesidades cada vez más elaboradas. En este desarrollo biológico hay un proceso de maduración, que es el resultado del proceso de mielinización de los axones de las neuronas. Por otro lado, Vygotsky y Wallon establecen una diferencia entre el desarrollo 
orgánico y el desarrollo cultural, el cual se refiere a todas las habilidades, saberes, valoraciones que la humanidad ha logrado obtener a lo largo de su historia. La interacción con el medio estimula a los pequeños a adquirir nuevos saberes de forma más eficaz y rápida.

Durante el primer año los niños empiezan a caminar, algunos lo logran un poco antes y otros demoran algunos meses más, no hay que alarmarse, los pequeños muestran mayor interés por realizar movimientos con todo su cuerpo, empiezan a empujar objetos o lanzarlos. Se muestran más independientes, intentan realizar algunas acciones solos, como por ejemplo, comer, colocar sus juguetes u objetos en cierto lugar.

Empiezan a manifestar su lenguaje comprensivo riendo ante alguna situación o frase o siguiendo alguna pequeña indicación, dirigen su mirada hacia quien los llama por su nombre, empiezan a realizar sonidos onomatopéyicos o hablar con palabras incomprensibles, es parte de la edad.

Pero si algún pequeño no logra realizar estas acciones, es importante que los padres y todas las personas encargadas de su cuidado, mantengan la calma y tengan presente que cada niño se desarrolla a su propio ritmo y debemos evitar comparaciones, las pautas de desarrollo solo muestran lo que el niño puede lograr; si no es en ese momento, dentro de un poco más de tiempo lo hará y mientras tanto se le puede ayudar, mediante ejercicios en forma de juegos. Por ejemplo, en el lenguaje, se debe tener en cuenta que muchos pequeños empiezan a hablar más, a partir de los dos años de edad y los podemos ayudar pronunciándoles adecuadamente los nombres de las cosas, enseñándoles a asociar los objetos con sus nombres, leyéndoles cuentos, mostrándoles las revistas de los supermercados, mencionando los objetos que están ilustrados, empezándoles a dar alternativas sobre alguna situación, como: preguntar si desea ponerse tal media u otra, puede que sorprenda con alguna respuesta.

Según Cardona, A. y Cardona, D. (2013), a los dos años el lenguaje se convierte en la herramienta de comunicación más eficiente, el niño comienza a desarrollar el sistema de aprendizaje articulatorio, comprende aproximadamente 500 palabras y reproduce mínimo 20, produce frases de mínimo 2 palabras y utiliza el "yo", "tu" y "mi", señala 4 o 5 partes del cuerpo, elabora preguntas, aprende canciones y aumenta la interacción en el juego con otros.

Según el Child Mind Institute (2014), otro tema no menos importante es el área sensorial, cómo el niño procesa la información a través de sus sentidos. Hay pequeños que sin tener un trastorno importante como es el autismo, presentan algunas dificultades sensoriales como por ejemplo poca tolerancia al ruido o poco control de sus impulsos, dificultad para probar alimentos nuevos o para tocar diferentes texturas u objetos, algunos muestran irritabilidad o no pueden conciliar un buen sueño. Este problema es importante abordarlo tanto en casa como con ayuda de terapistas ocupacionales, los cuales a través de diferentes actividades físicas diseñadas específicamente para poder regular esta información captada por los sentidos permitan que el niño que lo padece, poco a poco se vaya sintiendo más cómodo, seguro y capaz de realizar dichas actividades sin llegar a la irritabilidad. Aún no existen estudios científicos que respalden del todo las terapias ocupacionales.

En casa podemos ayudar con diferentes actividades, si el niño no controla mucho sus impulsos, hacerle más cariño, enseñarle a ser más delicado a través del ejemplo o si no le gusta las texturas, ayudarle a que poco a poco las vaya tolerando por medio del juego, a la hora de ingerir los alimentos, no limpiándolos inmediatamente y permitir de que se ensucien con la comida, tratar de buscar la manera de que el pequeño poco a poco vaya aceptando mancharse; por ejemplo, jugando con témperas, plastilina u otras masas, incentivándole a realizar diferentes actividades de motora fina. Si el problema es conciliar el sueño, bañarlos antes de dormir y realizarles masajes relajantes.

\section{Trabajo en aula}

El docente debe tener muy presente que cada alumno es una persona única, con su propia forma de ser y de sentir, con su propio ritmo de aprendizaje, es necesario que el maestro conozca 
a cada uno de sus niños, su historia familiar y sus capacidades, debilidades, fortalezas y necesidades. Que pueda trabajar en conjunto con los padres de familia y profesionales a cargo de ciertas necesidades del niño.

Debe planificar actividades que abarquen los tres estilos de aprendizaje y fomente la independencia de los alumnos brindándoles seguridad. Debe ser un guía, un orientador, ayudando a los alumnos a poder llegar a la respuesta por sí mismos. Sus clases deben ser significativas para los alumnos, debe tocar temas de interés que permitan que la clase se vuelva más interactiva y así pueda incentivar la crítica constructiva.

\section{CONCLUSIÓN}

Debemos observar siempre a nuestros pequeños, fomentar una adecuada autoestima, brindarles seguridad; siempre estimularlos y si presentan alguna dificultad evitar las comparaciones, brindarles más amor y un apoyo oportuno, respetando su grado de maduración cognitiva.

\section{REFERENCIAS}

De Zubiria, J. y colaboradores (2009). Los Ciclos en la Educación. Bogotá: Editorial Magisterio.

Russek, S. (2007). Crecimiento y Bienestar Emocional "Mejorando el Presente, Enriqueciendo el Futuro". Recuperado de http://www.crecimiento-y-bienestaremocional.com/autoestima-ninos.html

Guíainfantil.com "La autoestima y los niños". Polegar Medios S. L. 2000-2013. Recuperado de http://www.guiainfantil. $\mathrm{com} / \mathrm{salud} /$ autoestima/index.htm

Baby Center (2016). El desarrollo de tu hijo de 12 meses. Recuperado de http://espanol. babycenter.com/a2600094/el-desarrollo-detu-hijo-de-12-meses

Cardona, A.; Cardona, D. (2013). Desarrollo del Lenguaje Comprensivo y Expresivo en niños de 12 a 36 meses. CES Salud Pública.
Artículo de Investigación Científica. Recuperado de file:///C:/Users/jdorival /Downloads/Dialnet-DesarrolloDelLenguaje ComprensivoYExpresivoEnNinosD4890178.pdf

Muñoz, A. (2010). Psicología del Desarrollo en la Etapa de Educación Infantil. Madrid: Pirámide.

Child Mind Institute, socio fundador de Understood (2014) ¿Existen terapias o tratamientos para las Dificultades del Procesamiento Sensorial? Understood Dificultades de Aprendizaje y Atención. Recuperado de https://www.understood. org/es-mx/learning-attention-issues/childlearning-disabilities/sensory-processingissues/sensory-processing-disordertherapies-and-treatments 